\title{
Realistic MHD simulations of magnetic self-organization in solar plasma
}

\author{
I. N. Kitiashvili ${ }^{1,2}$, A. G. Kosovichev ${ }^{2}$, A. A. Wray ${ }^{1,3}$, and \\ N. N. Mansour ${ }^{1,3}$ \\ ${ }^{1}$ Center for Turbulence Research, Stanford University, Stanford, CA 94305, USA \\ email: irinasun@stanford.edu \\ ${ }^{2}$ W.W. Hansen Experimental Physics Laboratory, Stanford University, \\ Stanford, CA 94305, USA \\ ${ }^{3}$ NASA Ames Research Center, Moffett Field, Mountain View, CA 94040, USA
}

\begin{abstract}
Filamentary structure is a fundamental property of the magnetized solar plasma. Recent high-resolution observations and numerical simulations have revealed close links between the filamentary structures and plasma dynamics in large-scale solar phenomena, such as sunspots and magnetic network. A new emerging paradigm is that the mechanisms of the filamentary structuring and large-scale organization are natural consequences of turbulent magnetoconvection on the Sun. We present results of 3D radiative MHD large-eddy simulations (LES) of magnetic structures in the turbulent convective boundary layer of the Sun. The results show how the initial relatively weak and uniformly distributed magnetic field forms the filamentary structures, which under certain conditions gets organized on larger scales, creating stable long-living magnetic structures. We discuss the physics of magnetic self-organization in the turbulent solar plasma, and compare the simulation results with observations.
\end{abstract}

Keywords. Plasmas, turbulence, Sun: granulation, magnetic fields, sunspots, photosphere

\section{Introduction}

The interaction of turbulent plasma and distributed magnetic fields is presented by various phenomena on the solar surface. Modern observational instruments provide unique detailed data for analysis of the magnetic field topology and convective flows. However, observational limitations and complicated physics of the observed phenomena make of great interest the development of realistic numerical simulations. In these simulations a numerical model is built from first physical principles and takes into account the realistic equation of state, effects of ionization, chemical composition, radiative transfer, turbulence and magnetic field effects. The pioneering numerical investigations by Stein and Nordlund (1989) reproduced quite accurately physical properties of solar convection.

We investigate processes of self-organization of the solar convection in magnetic field by means of the realistic numerical simulations. Our numerical models reproduce the characteristic filamentary structurization and dynamics of magnetoconvection in the sunspot penumbra at different distances from the umbra, and also reveal a mechanism of spontaneous formation of long-living magnetic structures from initially uniform weak magnetic field in the upper convection. We used a 3D radiative MHD code, developed for simulations of top layers of the solar convective zone and low atmosphere (Jacoutot et al. 2008). Various sub-grid scale turbulent models are implemented in the code. Here we use a minimal hyperviscosity model. 

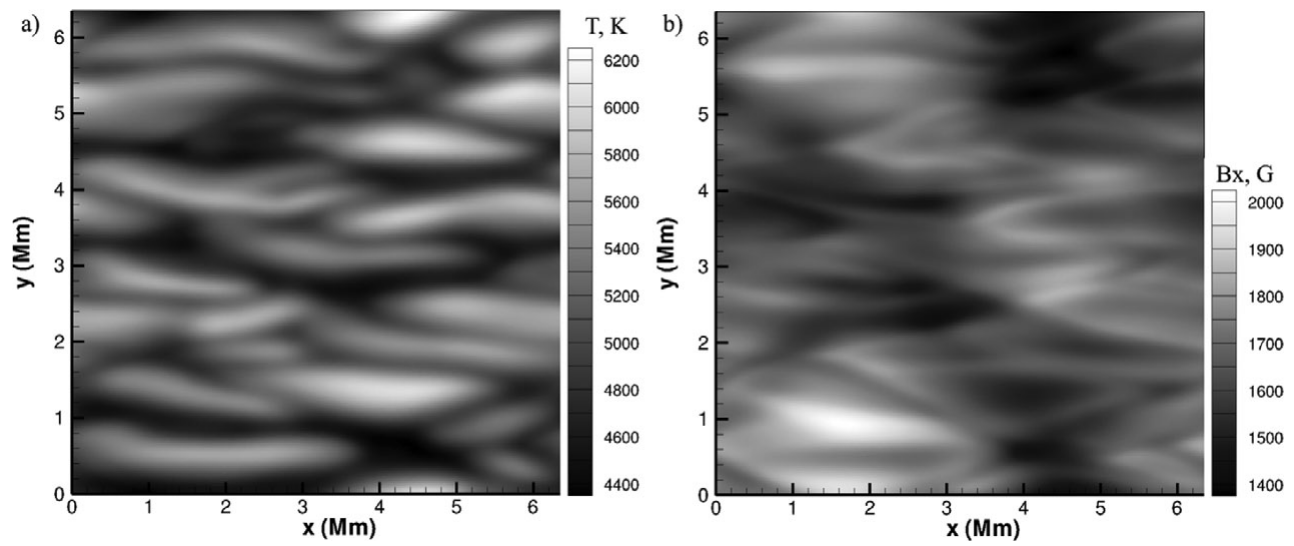

Figure 1. Filamentary structure of magnetoconvection at the solar surface in a highly inclined (along $x$-axis) strong magnetic field $\left(\mathrm{B}_{0}=1500 \mathrm{G}, \alpha=85^{\circ}\right)$ : a) distribution of the temperature surface, $\mathrm{T} ; \mathrm{b}$ ) horizontal component of magnetic field, $\mathrm{B}_{\mathrm{x}}$.

\section{Magnetic self-organization in a sunspot penumbra}

Magnetoconvection in a sunspot penumbra, where convective flows interact with strong, almost horizontal magnetic field, is a source of various observational phenomena. One of them is the Evershed effect, observed as a radial outflows of plasma along magnetic filaments with a speed of $2-6 \mathrm{~km} / \mathrm{s}$ (Evershed 1909). The highly organized behavior of the magnetized plasma flows indicates strong coupling between the dynamics of magnetic field and turbulent convection. The complicated radial distribution of the magnetic field in real sunspots creates difficulties in the numerical penumbra modeling. Therefore, for studying the basic physical properties we simulate separately different penumbra areas, in which the mean magnetic-field strength and inclination are approximately constant. Thus, the various magnetic field strengths, $600-2000 \mathrm{G}$, in our simulation domain, correspond to penumbra areas located at different distances from the sunspot umbra.

Our previous investigation of the influence of magnetic field inclination on solar granulation showed that the granular convective cells become deformed in the direction of the field inclination and form a dynamic filamentary structure of the magnetoconvection (Kitiashvili et al. 2009, 2010b). Such stretching of convective granules along the magnetic field lines becomes stronger with the higher field inclination to the surface and with the higher strength of magnetic field. In stronger magnetic field the convective filaments become more narrow, and this causes additional acceleration of the horizontal flows along the field lines. Figure 1 illustrates the surface distributions of temperature (panel a) and the horizontal component of magnetic field (panel b) for the simulations with the field mean strength of $1500 \mathrm{G}$ and the mean inclination angle of $85^{\circ}$ towards the surface. The thin elongated granules and the high-speed horizontal flows are located in the magnetized "gaps" with relatively low field strength. The surrounding strong magnetic field controls the direction of the flows and accelerates it by decreasing the space between the magnetic filaments. In addition, the solar convection in inclined magnetic field has properties of magnetoconvection waves traveling in the direction of the field inclination. This results in coherent patches of high-speed flows $(\sim 6 \mathrm{~km} / \mathrm{s})$, observed as "Evershed clouds". The patches appear quasi-periodically with a characteristic period of $20-40$ min. The coherent behavior of these flows across several filaments is particularly apparent in the strong field regions $(1500-2000 \mathrm{G})$. Such coherent behavior of the filamentary magnetoconvection represents an interesting example of non-linear self-organization of 

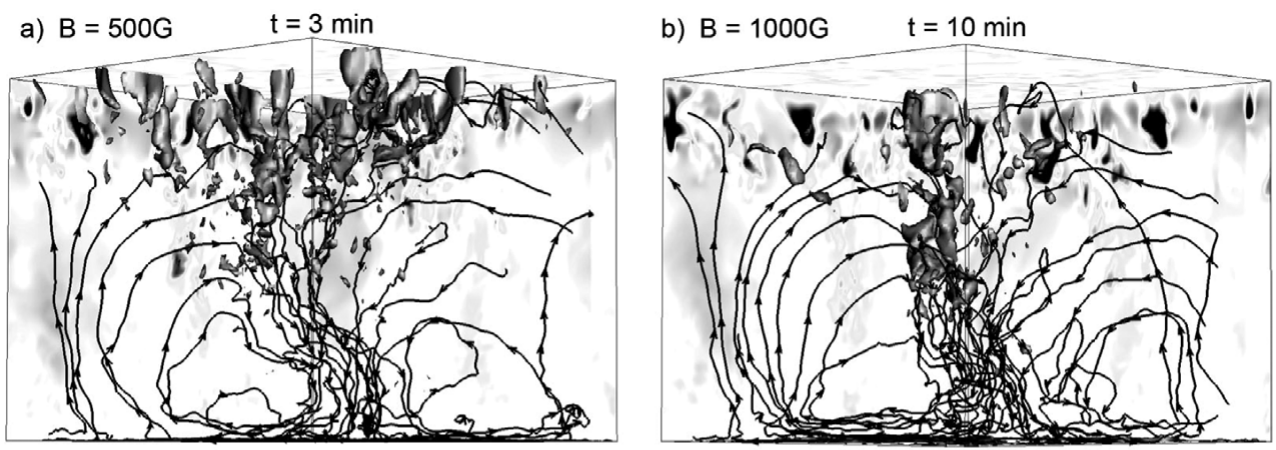

c) $B=1000 \mathrm{G} \quad \mathrm{t}=20 \mathrm{~min}$

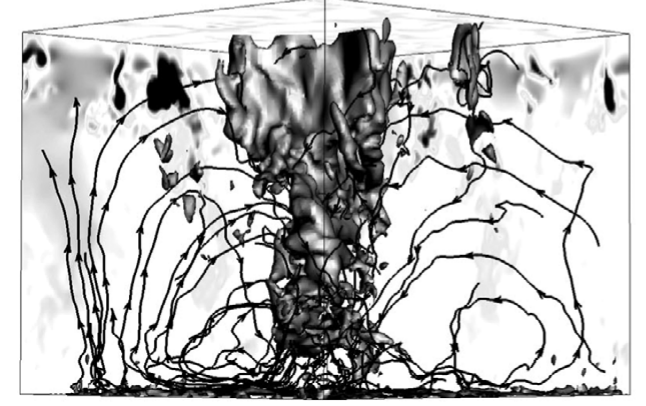

d) $B=1000 \mathrm{G} \quad \mathrm{t}=40 \mathrm{~min}$

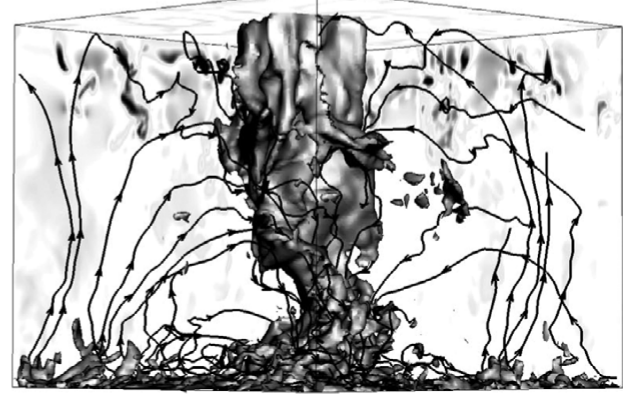

Figure 2. Process of formation of a magnetic structure in the subsurface layers of the Sun. The greyscale isosurface corresponds to $500 \mathrm{G}$ in panel a) and $1000 \mathrm{G}$ in panels b-d). Curves illustrate streamlines of converging subsurface flows.

turbulent magnetized plasma. The partially frozen magnetic field lines are deformed by dragging convective upflows and downflows. Such "sea-serpent" effect can be observed in the form of bipolar magnetic patches, which move in the direction of magnetic field inclination following the convective cell dynamics (Kitiashvili et al. 2010a).

\section{Effects of weak magnetic fields on the turbulent convection}

In regions with relatively weak ( $\ll 1000 \mathrm{G}$ ) magnetic fields on the Sun convection plays the lead role. The magnetic field gets organized by turbulent convective processes. For example, a weak initially uniform vertical magnetic field $\left(B_{0}=1-10 \mathrm{G}\right)$ becomes concentrated of the surface and near-surface layer in the intergranular lanes in the form of compact magnetic flux tubes (observed as bright points), in which the field strength reaches $\sim 1000 \mathrm{G}$ and which are accompanied downflows. In deeper subsurface layers this field forms diffuse magnetic structures. Thus, the MHD simulations show that the ubiquitous magnetic flux tubes observed in the "quiet"-Sun regions are highly dynamic and can be maintained in the near-surface layer by convective downdrafts.

For a stronger $\left(B_{0}=100 \mathrm{G}\right)$ initial vertical field, distributed uniformly in the simulation domain $\left(6.4^{2} \times 5.5 \mathrm{Mm}\right)$, our results reveal a process of magnetic field accumulation into a large-scale (pore-like) magnetic structure. This transient process starts in the vicinity of a large vortex tube $(\sim 0.5 \mathrm{Mm}$ in diameter $)$ after the uniform field initiation, and leads to creation of an unstable region. This region is characterized by a low gas pressure, strong downflows $(\sim 7 \mathrm{~km} / \mathrm{s})$ and supersonic horizontal velocities. The strong converging downflows and a sharp decrease of density cause further amplification of the magnetic field concentration in the region. 

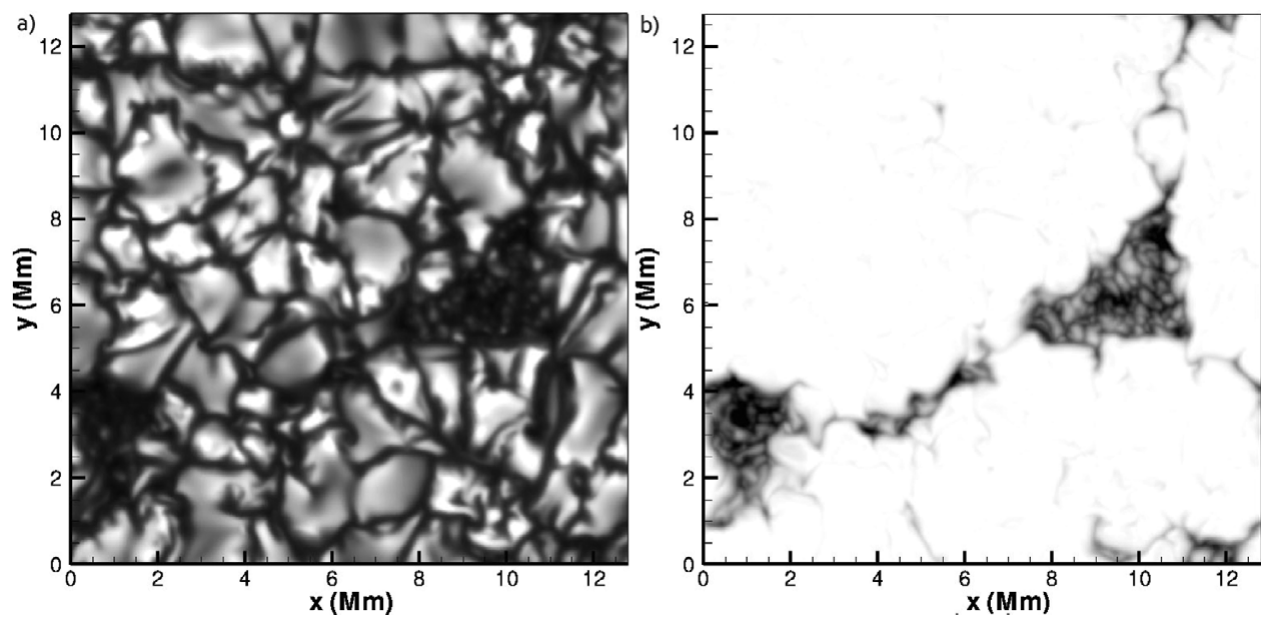

Figure 3. Formation of two magnetic structures in the simulations with a large box domain. The structures are shown at the surface in the blue continuum intensity (panel a) and the vertical magnetic field distribution (panel b), where the range of the grey scale is from 0 (white) to $1500 \mathrm{G}$ (black).

Figure 2 illustrates different stages of formation of the self-organized magnetic structure. The stream lines (with arrows) show converging convective flows that support the process of accumulation of magnetic field. During the first few minutes, the initial vertical uniform $100 \mathrm{G}$ vertical magnetic field is collapsed into the intergranular lanes. Locally the field strength increases up to $500 \mathrm{G}$ (shown as isosurfaces in Fig. 2a). Due to the converging flows and the local pressure cavity the individual magnetic patches move into the region of the structure formation (Fig. 2b). Finally, the complicated diffusive magnetic structure becomes compact and stable (Fig. 2c-d). The local accumulation of the magnetic field increases the magnetic field strength up to $\sim 1400-1800 \mathrm{G}$ at the surface, and up to $\sim 6 \mathrm{kG}$ below the surface (Kitiashvili et al. 2010c). It is interesting that this compact magnetic pore-like structure has long life-time, at least, for 9 hours computed in our simulations, but also it is highly dynamic and can be moved by occasional strong convective flows.

The process of the structure formation depends on the state of convection at the moment of magnetic field initialization. We repeated the simulations for the same domain $\left(6.4^{2} \times 5.5 \mathrm{Mm}\right)$, but for different moments of the magnetic field initialization and found that the self-organized structures can be formed in different places and have different properties, such as size, magnetic field strength and distribution with depth, but qualitatively the process is the same in all cases. In some cases, two separate magnetic structures with very different properties are formed. However, these structures are usually close to each other and merged during the evolution. In the case of a larger simulation domain $\left(12.8^{2} \times 5 \mathrm{Mm}\right)$ and a similar grid resolution $(25 \mathrm{~km}$ in horizontal and $22 \mathrm{~km}$ in the vertical directions), the uniformly distributed magnetic field became initially concentrated in five magnetic structures. During the evolution some of these structures merged, and finally we obtained two stable pore-like structures. Figure 3 shows an intensity image of these pores in the blue continuum (panel a) and also the corresponding vertical magnetic field map (panel b). An interesting feature of the intensity map is the internal substructure consisting of umbra-dot-like dots features inside the pores. The umbral dots represent small convective granules suppressed by the strong magnetic field. The distribution of the vertical magnetic field (Fig. $3 \mathrm{~b}$ ) shows that the pore-like structures are magnetically 
connected. Inside the structures, the magnetic field is mostly concentrated between the bright umbral dots.

\section{Conclusion}

Convection on the solar surface is characterized by strong turbulent processes. The influence of magnetic field makes the plasma flow behavior more organized. The almost horizontal magnetic field of sunspot penumbrae creates a subsurface shear layer, in which the plasma is accelerated in the direction of magnetic field inclination, leading to the Evershed effect. A weak vertical magnetic field (1 - 10 G) imposed in the convective layer results in formation of magnetic concentrations (bright points) with downdrafts at the solar surface, connected to diffuse subsurface magnetic structures. A stronger $100 \mathrm{G}$ field leads to a process of spontaneous formation of stable magnetic pore-like structures, with the magnetic field strength $\sim 1.5 \mathrm{kG}$ at the surface and $\sim 6 \mathrm{kG}$ below the surface. These examples of magnetic self-organization of turbulent convection on the Sun illustrate importance of the realistic numerical simulations, based on first principles, for getting insight into complicated non-linear phenomena of plasma astrophysics.

\section{Acknowledgement}

We thank the International Space Science Institute (Bern) for the opportunity to discuss these results at the international team meeting on solar magnetism.

\section{References}

Evershed, J. 1909, MNRAS, 69, 454

Jacoutot, L., Kosovichev, A. G., Wray, A., \& Mansour, N. N. 2008, ApJ, 682, 1386.

Kitiashvili, I. N., Kosovichev, A. G., Wray, A. A., \& Mansour, N. N. 2009, ApJ, 700, L178.

Kitiashvili, I. N., Bellot Rubio, L. R., Kosovichev, A. G., Mansour, N. N., Sainz Dalda, A., \& Wray, A. A. 2010a, ApJ, 716, L181.

Kitiashvili, I. N., Kosovichev, A. G., Wray, A. A., \& Mansour, N. N. 2010b, Proc. of 3rd Hinode Science Meeting, 1-4 December, 2009, in press.

Kitiashvili, I. N., Kosovichev, A. G., Wray, A. A., \& Mansour, N. N. 2010c, ApJ, 719, 307.

Stein, R. F. \& Nordlund, A. 1989, ApJ, 342, L95 